\title{
FUSION OF LIDAR DATA AND MULTISPECTRAL IMAGERY FOR EFFECTIVE BUILDING DETECTION BASED ON GRAPH AND CONNECTED COMPONENT ANALYSIS
}

\author{
S. A. N. Gilani ${ }^{\mathrm{a}, *}$, M. Awrangjeb ${ }^{\mathrm{b}}$, G. Lu ${ }^{\mathrm{b}}$ \\ ${ }^{\text {a }}$ Faculty of Information Technology, Monash University, Australia - alinaqi.gilani@ monash.edu \\ ${ }^{\mathrm{b}}$ School of Eng. and Info. Tech., Federation University Australia, Australia - \{mohammad.awrangjeb, guojun.lu\}@ federation.edu.au
}

Commission III, WG III/1

KEY WORDS: building, detection, segmentation, LiDAR, graph, NDVI, entropy, multispectral imagery

\begin{abstract}
:
Building detection in complex scenes is a non-trivial exercise due to building shape variability, irregular terrain, shadows, and occlusion by highly dense vegetation. In this research, we present a graph based algorithm, which combines multispectral imagery and airborne LiDAR information to completely delineate the building boundaries in urban and densely vegetated area. In the first phase, LiDAR data is divided into two groups: ground and non-ground data, using ground height from a bare-earth DEM. A mask, known as the primary building mask, is generated from the non-ground LiDAR points where the black region represents the elevated area (buildings and trees), while the white region describes the ground (earth). The second phase begins with the process of Connected Component Analysis (CCA) where the number of objects present in the test scene are identified followed by initial boundary detection and labelling. Additionally, a graph from the connected components is generated, where each black pixel corresponds to a node. An edge of a unit distance is defined between a black pixel and a neighbouring black pixel, if any. An edge does not exist from a black pixel to a neighbouring white pixel, if any. This phenomenon produces a disconnected components graph, where each component represents a prospective building or a dense vegetation (a contiguous block of black pixels from the primary mask). In the third phase, a clustering process clusters the segmented lines, extracted from multispectral imagery, around the graph components, if possible. In the fourth step, NDVI, image entropy, and LiDAR data are utilised to discriminate between vegetation, buildings, and isolated building's occluded parts. Finally, the initially extracted building boundary is extended pixel-wise using NDVI, entropy, and LiDAR data to completely delineate the building and to maximise the boundary reach towards building edges. The proposed technique is evaluated using two Australian data sets: Aitkenvale and Hervey Bay, for object-based and pixel-based completeness, correctness, and quality. The proposed technique detects buildings larger than $50 \mathrm{~m}^{2}$ and $10 \mathrm{~m}^{2}$ in the Aitkenvale site with $100 \%$ and $91 \%$ accuracy, respectively, while in the Hervey Bay site it performs better with $100 \%$ accuracy for buildings larger than $10 \mathrm{~m}^{2}$ in area.
\end{abstract}

\section{INTRODUCTION}

The building detection from remotely sensed data has become a topic of increasing importance as they are essential for a variety of applications in residential and urban areas. Their accurate boundaries are indispensable and significant for applications in the field of real estate, city planning, disaster management, cartographic mapping and civilian and military emergency responses (Sohn and Dowman, 2007, Li and Wu, 2013). The automatic boundary extraction is challenging due to building shape variability and surrounding environment complexity. High resolution imagery contains rich spectral information that is suspectable to noise and can easily be affected by contrast, illumination, and occlusion. Under certain conditions, similar objects may appear with varying spectral signatures while different objects may provide co-spectral signatures ( $\mathrm{Li}$ and $\mathrm{Wu}, 2013$ ).

The airborne Light Detection and Ranging (LiDAR) can rapidly acquire high-precision three-dimensional information of largescale areas by emitting and receiving the laser pulses. The height variation is a more suitable cue for detecting elevated objects and delineating building boundaries more than the spectral and texture changes. However, the horizontal accuracy of boundaries extracted from LiDAR data is poor, which makes detection more difficult (Chen and Zhao, 2012, $\mathrm{Li}$ and $\mathrm{Wu}, 2013$ ). The urban areas that are characterised by complex scenes, the appearances

\footnotetext{
*Corresponding authors: alinaqi.gilani@monash.edu
}

of trees and buildings in the LiDAR data might be similar (Rottensteiner et al., 2005). The combination of these two paradigms promises to produce results at greater accuracy than the contributions of either field alone. Therefore, many researchers have been attempting to integrate multi-source data to detect buildings in urban areas.

According to Lee et al. (Lee et al., 2008), building detection approaches can be broadly categorised into three distinct groups. The first group contains methods that employ only 2D or 3D information from photogrammetric imagery to detect buildings and discern from surrounding objects (Mayer, 1999). The complexity of these techniques increases proportionally with the increase in richness of information in high-resolution imagery, e.g. derivation of depth information from stereo to effectively address occlusion and shadow factors (Yong and Huayi, 2008). In addition, nearby trees of similar height also make the use of such derived range data difficult (Lee et al., 2008).

The second group ensembles the techniques that attempt to detect building regions from LiDAR data by classifying the data points into ground and non-ground data based on elevation (Lee et al., 2008). It is reported that LiDAR offers an improved level of automation in the building detection process when compared to image-based detection algorithms (Vu et al., 2009). On the contrary, Oude Elberink (Elberink, 2008) discusses the issues upfront in LiDAR based building detection techniques and shows the effect of raw interpolated data usage on the performance of detection process (Demir et al., 2009). Moreover, the horizontal 
accuracy of boundaries extracted from LiDAR data is poor because of laser pulse discontinuity ( $\mathrm{Li}$ and $\mathrm{Wu}, 2013)$ and hard to obtain geometrically precise boundary using only LiDAR points cloud (Yong and Huayi, 2008). The quality of regularised building boundaries also depends on LiDAR resolution (Sampath and Shan, 2007).

LiDAR generally provides more accurate height information but lacks in horizontal accuracy whereas high-resolution imagery entails precise horizontal accuracy. The third category of methods exploits the complementary benefits of both LiDAR data and photogrammetric imagery. More specifically, laser intensity and height information in LiDAR data can be used along with texture and region boundary information extracted from aerial imagery to improve detection accuracy (Lee et al., 2008). As a result, several authors promoted the fusion of two data sources as a promising strategy to extract high quality building boundaries (Rottensteiner et al., 2005, Yong and Huayi, 2008, Demir et al., 2009, Li and $\mathrm{Wu}, 2013)$.

However, it is still challenging to extract the correct and relevant features from multispectral image and LiDAR data of a particular area to detect buildings. But how to amalgamate the respective data sources such that their weaknesses can be compensated effectively is a question that requires further investigation. Additionally, there is no rule of thumb to integrate different features for automatic building boundary extraction of various building shapes: curved, wavy, zigzag, and other irregular structures. Most existing fusion methods can only handle simple building shapes like polygons (Yong and Huayi, 2008, Awrangjeb et al., 2010).

Currently, there are no unanimous evaluation system and standard guidelines available to measure the performance of building extraction techniques (Rutzinger et al., 2009, Awrangjeb and Fraser, 2014a). Indeed, evaluation results are often missing from published accounts of building detection; the use of 1-2 evaluation indices only has characterised many studies (Demir et al., 2009, Awrangjeb et al., 2010). This research aims to develop, firstly, a mechanism to effectively integrate features extracted from $\mathrm{Li}$ DAR and multispectral imagery to increase building detection performance and, secondly, a comprehensive evaluation of the proposed method using an automatic performance evaluation system using 15 evaluation indices (Awrangjeb and Fraser, 2014a).

In order to automatically detect buildings from complex geographic environment and delineate the boundaries, a new method is proposed that integrates both airborne LiDAR and multispectral imagery. The raw LiDAR data is divided into ground data and nonground data based on height information from bare-earth DEM and create a primary building mask. All the strongly connected pixels, the black pixels, present in the primary building mask are cumulated into contiguous regions that are later processed to estimate initial boundary and component labelling. The resultant information is further utilised to construct a disconnected components graph where each subgraph corresponds to a contiguous region of black pixels. The resultant disconnected graph may contain heaps of subgraphs due to the fact that even a single isolated black pixel from primary mask become a subgraph with one node. The subgraphs identified by this process along with respective boundaries and labels are further processed.

The image lines are extracted and classified using (Awrangjeb et al., 2013) and fed into a graph-based clustering procedure. The clustering process aims to associate the line segments to the corresponding subgraph using Dijkstra algorithm. The subgraphs that fail to cluster any line are eliminated for subsequent processing and, thus, reduce the initial count of prospective building objects. In order to eliminate vegetation and detect building objects, the input image is segmented into homogeneous grids and then individual cells are accumulated based on NDVI, entropy, and height difference. This process detects building objects, separates the occluded building parts from vegetation, and estimate the respective boundary. Finally, the building boundary is expanded, if possible, by accumulating the pixels based on its NDVI, entropy, and neighbouring LiDAR points cloud. It is experimentally demonstrated that the proposed technique can detect polyhedral buildings with a favourable success rate. We achieved above $90 \%$ detection rate in densely vegetated Australian urban environments whereas $100 \%$ in a low vegetation area.

The involved automatic evaluation system employs both objectand pixel-based indices. Although pixel-based evaluation reflects the horizontal accuracy, but the geometric evaluation is also used as a means of direct estimation of geometric accuracy.

\section{RELATED WORK}

The integration of high-resolution imagery and LiDAR data is well appreciated as it yields complementary benefits by providing a more complete scene description enriched with both spectral and 3D surface information. This integration of both data sources has been exploited to increase the classification performance and to improve the accuracy and robustness in automatic building detection, reconstruction and change detection techniques (Awrangjeb et al., 2010, Awrangjeb et al., 2012, Li and Wu, 2013).

These integration techniques can be categorised into two subsequent groups. Firstly, the integration techniques reported in literature (Sohn and Dowman, 2007, Lee et al., 2008, Demir et al., 2009, Awrangjeb et al., 2010, Awrangjeb et al., 2012, Chen and Zhao, 2012, Awrangjeb et al., 2013, Li and Wu, 2013) utilise both the LiDAR data and the imagery as the primary cues to delineate building outlines. They also employ the imagery to extract features like NDVI, entropy, shadow, and illumination in order to eliminate vegetation. Consequently, they offer better horizontal accuracy for the detected buildings. Our proposed building detection technique falls under this group. (Rottensteiner et al., 2003) generates two digital surface models (DSMs) from the first and last pulse return of the LiDAR data, which are used along with NDVI for the detection process. A morphological filter is applied over the last pulse DSM to generate a digital terrain model (DTM). The initial building regions are identified based on height, size, NDVI and the difference between the first and last phase of the DSMs.

The research reported in (Awrangjeb et al., 2010) uses LiDAR to generate DEM in order to separate non-ground and ground data points. Two masks are generated to identify the elevated area, which is subsequently used to extract line segments. The line segments formed around trees are removed using NDVI while remaining line segments are used to detect initial building positions. Finally, building boundaries are obtained after boundary expansion process using YIQ colour system. The article (Chen and Zhao, 2012) uses normalised DSM (nDSM) to remove the ground objects according to a height threshold. Keeping in view the relief displacement effect and the overlap ratio to avoid overremoving, nDSM is segmented by the region-growing method. Finally, the region size and spatial relation of trees and buildings are used to filter out trees occluded by buildings based on an object-based classification. Another technique aiming at both detection and reconstruction (Awrangjeb et al., 2013) extracts initial building position from LiDAR using height information from DEM. Later, the line segments are extracted, classified and further used in region growing technique to obtain building planes 
followed by building boundary. Vegetation is removed using features such as NDVI, entropy and height information from optical image and LiDAR data.

Secondly, there are techniques, which use the LiDAR data as the primary cue for building detection and use the features from optical imagery only to remove vegetation (Vu et al., 2009, Rottensteiner et al., 2005). Consequently, these methods suffer from poor horizontal accuracy for the building boundary delineation. The method details in (Rottensteiner et al., 2005) uses the Dempster-Shafer theory to classify LiDAR data points into constituent groups: buildings, trees, grassland or bare soil. However, the reported detection performance is adversely affected for small sized buildings (Rottensteiner et al., 2007). The reason lies if the Dempster-Shafer model remains untrained, the misclassification rate increases considerably (Khoshelham et al., 2008). Vu et al. (Vu et al., 2009) uses a morphological scale space for extracting building footprints from the elevation data and then remove vegetation using the spectral information. The detection performance was low and high computational complexity was reported because of using the scale space.

The method presented in this research is fully data-driven and self-adaptive for diverse building shapes. This paper is organised as follows. The work flow and detailed procedure of the proposed method is provided in Section 3. The evaluation system and experimental results are described and discussed in Section 4. Finally, the conclusion and future research direction are provided in Section 5.

\section{PROPOSED METHODOLOGY}

The work flow of the proposed method is shown in Figure 1. The labels from 1-6 describe the order of sub-processes towards building boundary delineation. The first step, blue coloured dashed rectangle, is a data preprocessing phase where we separate LiDAR points cloud, generate DEM, compute image entropy, NDVI and extract image line segments. The proposed method first divides the input LiDAR data into ground and non-ground points. The non-ground points, representing elevated objects above the ground such as buildings and trees, are further processed for building detection.

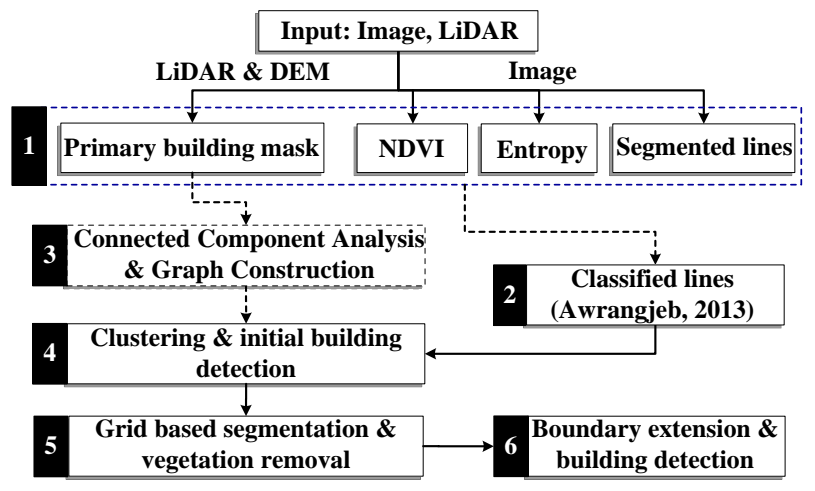

Figure 1. Work flow of proposed building detection technique

During the second phase, the extracted image lines are classified into several classes e.g. ground, ridge, and edge. The line segments that belong to "edge" and 'ridge" classes are of interest because these lines are either close or fall within the area of elevated objects. The next phase begins with Connected Component Analysis (CCA) and graph construction, where all the strongly connected pixels from the primary building mask are collected into individual components followed by initial boundary extraction. Moreover, we construct a disconnected components graph from connected components that clusters the classified lines to their particular components. We employ Dijkstra shortest path algorithm to establish the relevance of a segmented line to its corresponding component extracted from the primary building mask. The connected components that fail to cluster any line during clustering process are eliminated for further investigation, which reduces actual objects found at the first place.

We establish from literature that average height difference between neighbouring LiDAR points on building rooftops change constantly but on trees, height variation is abrupt and changes variably. Moreover, the NDVI and entropy measures are relatively high on vegetation than building roofs (Awrangjeb et al., 2013). Therefore, to eliminate the vegetation and detect buildings, which may partly occluded or non-occluded, the multispectral image is divided into equi-sized grids. The grid cells are accumulated under certain criteria for detection and elimination purposes. The grid-based segmentation process detects the buildings and estimate their corresponding boundaries. The boundary of detected buildings are finely delineated but are much shrinker due to misregistration between LiDAR and corresponding multispectral image. Therefore, the obtained boundaries are processed and are expanded, if possible, to extend its coverage towards building edges by accumulating individual pixels based on NDVI, entropy, and LiDAR data.

The input image in Figure 2(a) is an urban site, Aitkenvale (AV), in Queensland Australia that covers an area of $214 \mathrm{~m}$ x $159 \mathrm{~m}$ and contains 63 buildings. In the following subsections, different steps of the proposed method are described using this scene.

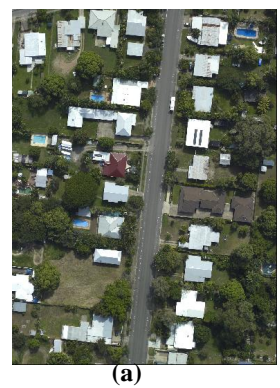

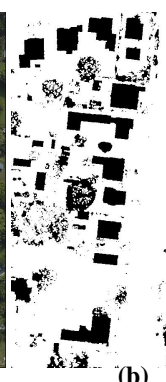

(b)

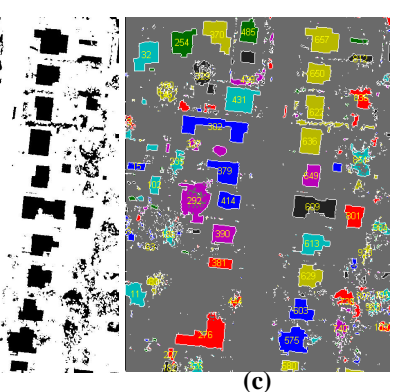

(c)
Figure 2. Sample data: (a) Aitkenvale multispectral image, (b) Primary building mask, and (c) CCA output with initial boundary and labels

\subsection{DATA PREPROCESSING AND LINE SEGMENTATION}

We generate a bare-earth DEM with $1 \mathrm{~m}$ resolution from input LiDAR data. The bare-earth DEM is also named as Digital Terrain Model (DTM) but we refer as DEM. A primary building mask is generated, see Figure 2(b), using LiDAR data and height information from DEM according to the method reported in (Awrangjeb et al., 2010). A height threshold is computed for each LiDAR point as $H_{t}=H_{g}+H_{r f}$, where $H_{g}$ is ground height and $H_{r f}$ is a relief factor that separates low objects from higher objects. For our study, we choose $1 \mathrm{~m}$ relief factor in order to keep low height objects (Awrangjeb and Fraser, 2014b).

Additionally, NDVI is calculated for each image pixel position from the input image that can either be a multispectral orthoimage or RGB colour image. If multispectral orthoimagery is not 
available, then the pseudo-NDVI is calculated from a colour orthoimage (Awrangjeb et al., 2013). The texture information like entropy is estimated at each image pixel location using a greyscale version of the image(Gonzalez et al., 2003). In step 2 of Figure 1 , the same grey-scale image is used to extract line segments, which are classified into several groups: ground, edge, and ridge lines, using the reported method in (Awrangjeb et al., 2013). The classification of line segments into groups is performed based on NDVI, entropy, and primary building mask computed in preceding phase. The segmented lines that belong to 'edge" and 'ridge" classes are displayed in Figure 3(c).

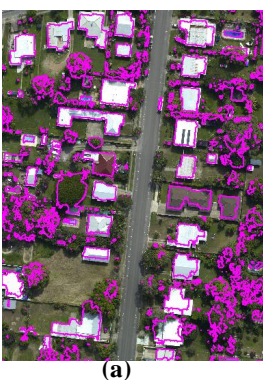

(a)

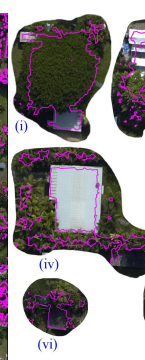

(b)

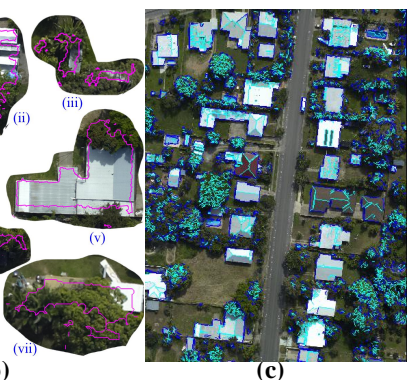

Figure 3. (a) Building boundaries detected after CCA, (b) False boundary delineation, and (c) Segmented lines (edges and ridges)

\subsection{INITIAL DETECTION USING CONNECTED COM- PONENT ANALYSIS AND GRAPH TECHNIQUE}

A connected component of value $v$ is a set of pixels $\xi$ where each pixel having a value $v$, such that every pair of pixels in the set $\phi$ are connected with respect to $v$. Provided that the primary mask in Figure 2(b) is a binary image that contains only two colours: black and white, where black regions describe the elevated areas. So, $P(r, c)=P\left(r^{\prime}, c^{\prime}\right)=v$ where either $v=$ black or $v=$ white. The pixel $(r, c)$ is connected to the pixel $\left(r_{0}, c_{0}\right)$ in 8neighbourhood connectivity with respect to value $v$ if there is a sequence of black pixels $(r, c)=\left(r_{0}, c_{0}\right),\left(r_{1}, c 1\right) \ldots\left(r_{n}, c_{n}\right)=$ $\left(r_{0}, c_{0}\right)$ where $P\left(r_{i}, c_{i}\right)=v ; \mathrm{i}=0 \ldots n$, and $\left(r_{i}, c_{i}\right)$ neighbours $\left(r_{i-1}, c_{i-1}\right)$ for each $\mathrm{i}=1 \ldots n$. The sequence of pixels $\left(r_{0}, c_{0}\right)$. forms a connected path from $(r, c)$ to $\left(r_{0}, c_{0}\right)$.

The CCA process detected 936 different objects from the primary building mask. Subsequently, we also estimated the boundary of each component using the Moore-Neighborhood tracing algorithm. The output of CCA process is shown in Figure 2(c), where the detected objects are labelled and plotted with different colours as of their neighbours. To visually inspect the building outline accuracy, the estimated boundaries are shown in Figure 3(a). We can clearly notice that few buildings are well delineated but several regions are formed on trees due to elevation. We can further observe that various buildings are occluded partly or fully by nearby vegetation. Moreover, some buildings are connected to each other by dense vegetation giving an indication of a single object. The building objects displayed in Figure 3(b) from (i)(vii) describe some such situations.

So, we use the segmented lines as a feature of structural objects to eliminate the false components formed on vegetation. The lines on vegetation are differentiable because they are discontinuous, smaller, and does not establish any geometric relationship - parallel, perpendicular, or diagonal relationship. Furthermore, we use 'edge' and 'ridge' lines because they are either close to the boundary or fall completely within the boundary of a particular object. In order to establish the relationship among lines and the association with their corresponding objects, we construct a bidirectional disconnected components graph, where each connected component from the preceding stage is represented as a subgraph. Each pixel of an object corresponds to a node where its 8-neighbouring pixels become its child nodes. The edge from a node to its descendent is assigned a unit weight. On the contrary, there is no path between black-pixel to white-pixel and vice versa. Therefore, no edge/path is represented by "inf" edge weight to differentiate between valid and invalid paths.

The image lines clustering process begins with the selection of a longest 'ridge" line as a centroid that falls within the boundary of a particular object. Then, we compute the shortest distance between centroid and surrounding lines using Dijkstra algorithm. The situation when the candidate line is along or on a particular shape/object, we always obtain a valid path of some weight otherwise the path weight is infinite indicating that the candidate line does not belong to the current cluster. We continue this process until all objects detected from the primary building mask are processed. The objects that fail to cluster any line are eliminated for further investigation. In this case, many objects that are formed on trees due to few LiDAR points are successfully removed and the number of total potential objects decreased to 307 from an initial count of 936 .

\subsection{VEGETATION REMOVAL AND BOUNDARY DELIN- EATION}

A visual comparison between Figure 3(a) and 4(a) reveals that clustering procedure drastically reduces the number of objects identified previously without losing any potential objects including small and low height buildings. But still it is observable that heaps of false objects/boundaries exist on trees and even outline of large buildings are not properly delineated. We can further notice in Figure 4(a) labelled from (i) to (ix) and their corresponding zoomed version in Figure 4(b) that there are various buildings, which are heavily occluded by dense vegetation.
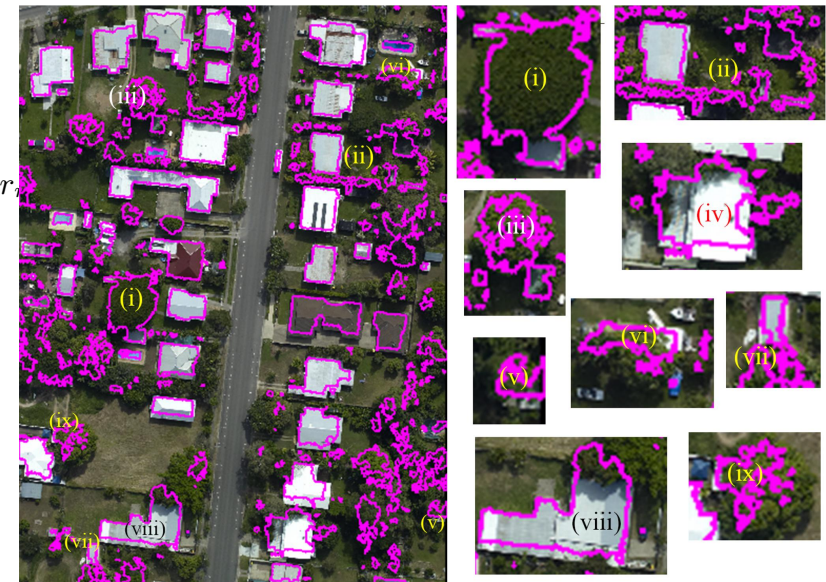

Figure 4. (a) Building after clustering process, (b) Occluded and false delineated buildings

From literature, we know that LiDAR height difference on polyhedral surfaces remains uniform and changes constantly. But in case of vegetation, LiDAR points reflect back from different height levels, therefore, the height changes in neighbouring LiDAR points are quite sudden and variable. In addition to height difference cue, NDVI and entropy measures have been extensively used in literature to eliminate the vegetation. However, to remove vegetation and, thereby, to recover the occluded buildings, the input image is segmented into regular sized grid to delineate the building boundary. The grid size is indirectly proportional to LiDAR point density, higher the density tighter can be 
the grid size. The grid size is chosen keeping the fact that each grid cell must contain LiDAR points. For example, in case of $\mathrm{AV}$ data set, LiDAR point density is 29 points $/ \mathrm{m}^{2}$ that allows to choose a fairly smaller grid size of $25 \mathrm{~cm}$. Therefore, the test scene is divided into rows and columns of the selected grid size.

It is quite evident from Figure 4(a) that the building objects identified are not well delineated, boundaries are over-segmented and, in few cases, see (i)-(iii) and (vi) in Figure 4(b), the prospective building objects are heavily occluded, and are mutually connected. To cope with the issues surfaced, each identified object is processed separately for vegetation removal, building recovery, and its respective boundary delineation.

Firstly, the coarse boundary associated with an object is utilised to collect the grid cells that creates a stack. Then a cell from top of the stack is selected, called seed cell, followed by the selection of cells in its 8-neighbourhood. Each neighbouring individual cell that exhibits similar height difference, NDVI, and entropy value with respect to the seed cell is accumulated, and thus, forms a group. The seed and its neighbouring cells, which meet the condition, are removed from the stack, and pushed into a priority queue. The seed element is marked to avoid for further selection and a new seed cell is chosen from the queue followed by its neighbourhood from the stack. All candidate cells are again evaluated for different matrices: height difference, NDVI, and entropy, and later pushed into the queue. This cell-based segmentation process continues until the last cell remains in the stack. The cells, which fail to form a group are eliminated during execution.

This process engenders contiguous groups of cells that are formed within the object's boundary as shown in sub-figures labelled (i)(vii) in Figure 5(a). It can be seen in the top left image labelled (i) that cell-based segmentation process has formed several groups, among all, two groups are formed on real building objects. Finally, a rule-based procedure is adopted that is based on LiDAR points height difference, NDVI, and entropy, the cumulated cells, which are confined within the boundary, are eliminated and two occluded buildings are recovered. The final detected buildings and their corresponding boundaries are shown 'yellow' in colour filled with red, while the groups/clusters marked for elimination have no boundary around.
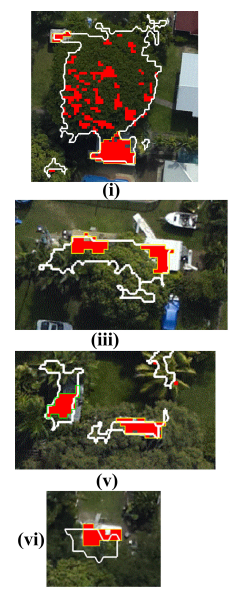
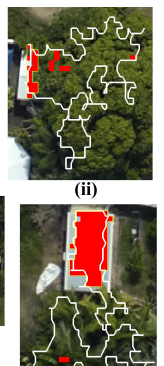

(iv)

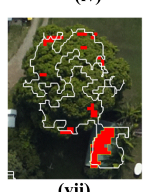

(vii)

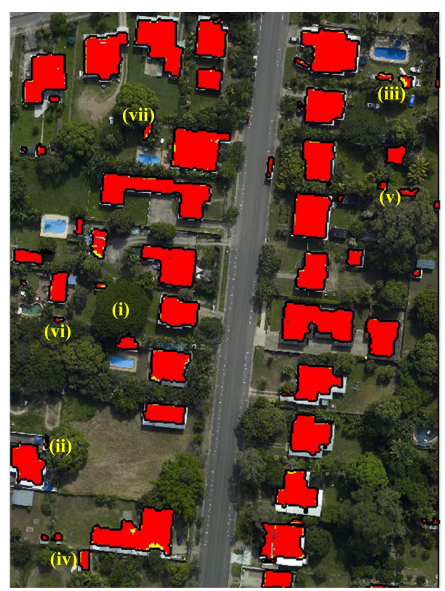

Figure 5. (a) Vegetation removal and recovery of occluded buildings, (b) Detected buildings

The same operation is performed for all the building objects extracted from the last phase. It can be observed in Figure 5(a), all buildings labelled from (ii) to (vii) are well delineated and cover the actual building region. The outcome of this procedure can be seen in Figure 5(b), where false buildings have been removed, occluded buildings are recovered and building boundaries are well delineated.

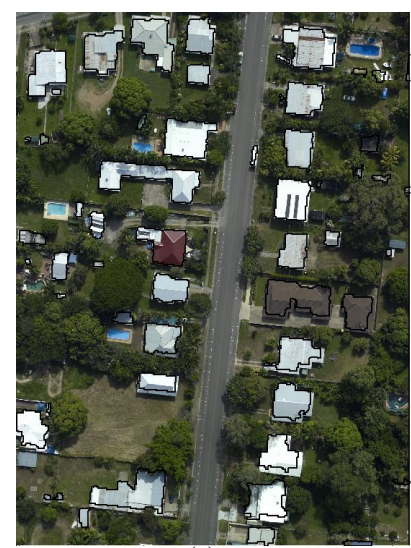

(a)

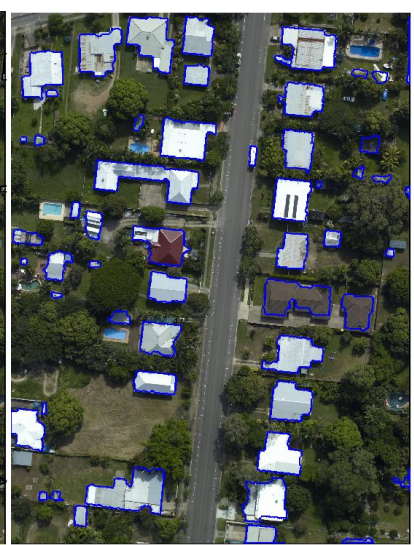

Figure 6. (a) Buildings before boundary expansion, (b) Final detected buildings

The boundaries of finally detected buildings in Figure 5(b) and Figure 6(a) are well outlined but they sometimes lack in a complete rooftop coverage due to large grid size as compared to the image resolution. Similar to the grid-based segmentation process, we accumulate pixels into boundary instead of cells and grow the boundary region towards edges for accurate delineation of the building boundary. For pixel based extension procedure, we first determine all the corresponding pixels of a building boundary and grow the boundary pixels in 8-neighbourhood fashion. For all new pixels, which does not lie within the already established boundary, we compute the pixel NDVI and height of their respective LiDAR data points. The pixel is included as a new boundary pixel based on comparison with average NDVI of pixels and LiDAR points average height within the boundary. Similarly, rest of the boundary pixels including new pixels, if any, are processed. We repeat the same process for all the buildings. The pre-expansion and post-expansion boundaries can be seen in Figure 6(a) and Figure 6(b) respectively. The later figure shows the final building boundaries that cover the building rooftop well close towards the building edges and will be used for evaluation purpose.

\section{EXPERIMENTAL RESULTS AND DISCUSSION}

To evaluate the performance of the proposed approach, two test data sets from two different sites are used. The objective evaluation follows a modified version of a previous automatic and threshold-free evaluation system (Awrangjeb and Fraser, 2014a).

\subsection{DATA SETS}

The test data sets as shown in Figure 7 cover two urban areas in Queensland, Australia: Aitkenvale (AV) and Hervey Bay (HB). The AV data set has a point density of 29 points $/ \mathrm{m}^{2}$ and comprises of a scene that covers an area of $214 \mathrm{~m} \times 159 \mathrm{~m}$. This scene contains 63 buildings, out of those four are between 4 to $5 \mathrm{~m}^{2}$ and ten are between 5 to $10 \mathrm{~m}^{2}$ in area. The HB data set has one scene and covers $108 \mathrm{~m} \times 104 \mathrm{~m}$ and contains 25 buildings. Both of these data sets contain mostly residential buildings and can be characterised as urban with medium housing density. The HB scene has low tree coverage that partially covers buildings but the AV site has densely vegetation and heavily occluded buildings. In terms of topography, both the sites are flat. 

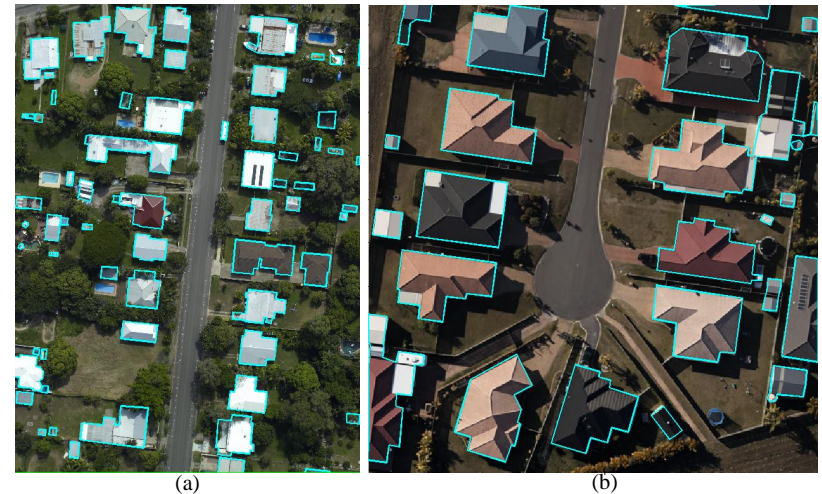

Figure 7. Data sets with reference boundary: (a) Aitkenvale, (b) Hervey Bay

\subsection{EVALUATION SYSTEM}

In order to assess the performance of the proposed method, the automatic and threshold-free evaluation system (Awrangjeb and Fraser, 2014a) has been employed. It offers more robust objectbased evaluation of building extraction techniques than the thresholdbased system (Rutzinger et al., 2009) adopted by the ISPRS benchmark system. It makes one-to-one correspondences between detected and reference buildings using the maximum overlaps, estimated by number of pixels. If a reference building $r_{r}$ and a detected building $r_{d}$ overlaps each other and they do not overlap any other entities, a true-positive (TP) correspondence is simply established. If a detected building $r_{d}$ does not overlap any $r_{r}$, then it is marked as a false positive (FP). Similarly, if a reference building $r_{r}$ does not overlap any $r_{d}$, then it is marked as a false negative (FN). In any other cases, an $r_{d}$ overlaps more than one $r_{r}$ and/or an $r_{r}$ overlaps more than $r_{d}$. In these two cases, a topological clarification is executed to merge or split the detected entities.

The reference data sets overlaid on the two test data sets, as shown in Figure 7, are used. The objective evaluation uses different evaluation metrics for three different types of evaluation categories: object-based, pixel-based, and geometric. For object-based metrics, completeness, correctness, quality, under- and over-segmentation errors give an estimation of the performance by counting the number of detected objects in the study. Whereas pixel-based metrics such as completeness, correctness and quality measures determine the accuracy of the extracted objects by counting the number of pixels. In addition, the geometric metric, root mean square error (RMSE), indicate the accuracy of the extracted boundaries with respect to the reference entities. Moreover, the number of over- and under-segmentation cases are estimated by the number of split and merge operations required during the topological clarification.

The minimum areas for large buildings and small buildings have been set to $50 \mathrm{~m}^{2}$ and $10 \mathrm{~m}^{2}$, respectively, in Awrangjeb and Fraser (Awrangjeb and Fraser, 2014b). Thus, the object-based completeness, correctness and quality values will be separately shown for large buildings and small buildings.

\subsection{EVALUATION RESULTS}

Figures 8 and 9 show the extracted buildings for the AV and HB data sets. The preceding figure also shows some complex cases, where the proposed method has not only detected small buildings but has also recovered the occluded buildings successfully.

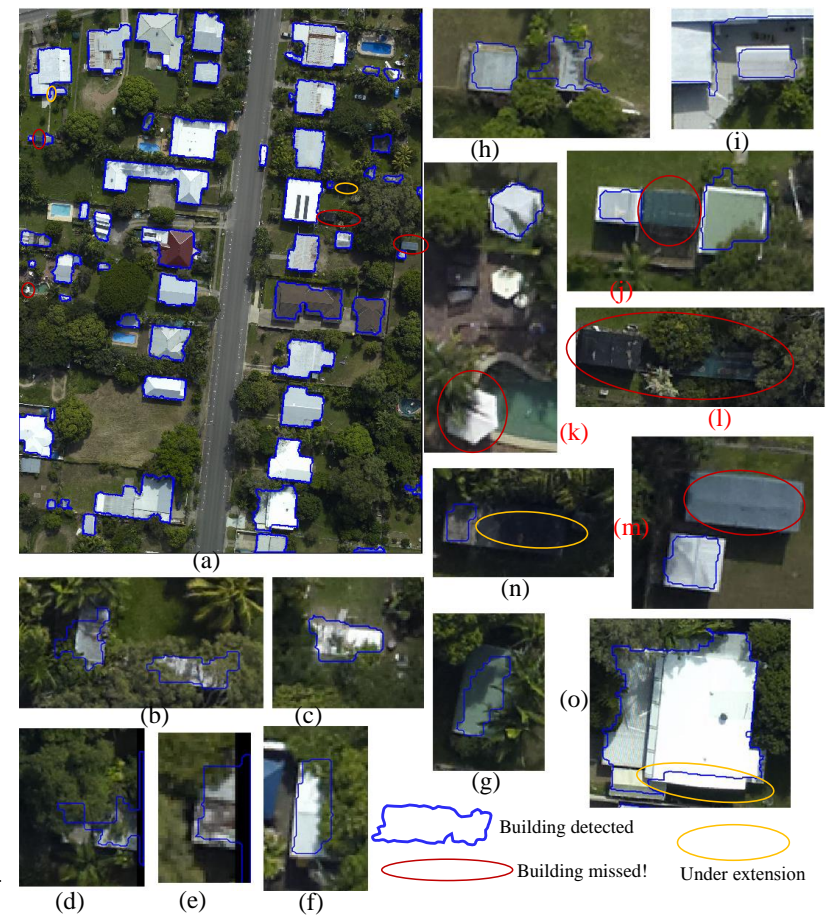

Figure 8. (a) Building detection on AV data set, (b)-(g) Magnified version of the buildings detected under complex scene, (h)-(i) Boundary over-extension, (j)-(m) Undetected buildings, (n)-(p) Boundary under-extension

For quantitative analysis of proposed method, object-based evaluation results are presented in Table 1, while pixel-based and geometric evaluation results are given in Table 2 , respectively.

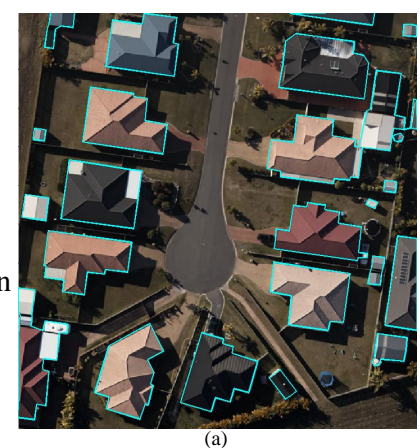

(a)

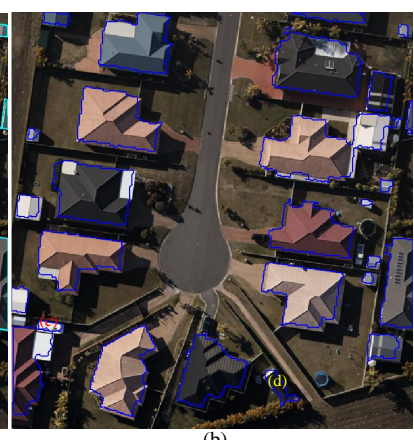

(b)
Figure 9. (a) Hervey Bay reference data set, (b) Detected buildings

The proposed technique is equally effective in both test areas. If we consider all reference buildings irrespective of their areas, the average completeness and correctness in object-based evaluation is above $92 \%$ with an average quality of about $92 \%$ (Table 1). All the buildings detected from Aitkenvale test scene can be seen in Figure 8(a). The magnified figures from (b) to (g) show some complex cases, where buildings are completely occluded by nearby dense vegetation. Two such buildings can be observed in Figure 9(b). Building (d) is of equal height of the surrounded vegetation and building (c) is close enough to a large building.

The sub-figures (h) and (i) in Figure 8 show the cases where over extension of building boundaries can be observed. This phenomenon occurred due to misregistration between LiDAR data and the corresponding multispectral image. During the pixel based 


\begin{tabular}{lccccccccc}
\hline Areas & $C_{m}$ & $C_{r}$ & $Q_{l}$ & $C_{m, 50}$ & $C_{r, 50}$ & $Q_{1,50}$ & $C_{m, 10}$ & $C_{r, 10}$ & $Q_{1,10}$ \\
\hline \hline AV & 84.62 & 100 & 84.62 & 100 & 100 & 100 & 91.67 & 100 & 91.67 \\
HB & 100 & 100 & 100 & 100 & 100 & 100 & 100 & 100 & 100 \\
\hline Avg & 92.31 & 100 & 92.31 & 100 & 100 & 100 & 95.83 & 100 & 95.83 \\
\hline \hline
\end{tabular}

Table 1. Building detection results: object-based evaluation for the Aitkenvale $(A V)$ and Hervey Bay $(H B)$ data sets in percentage. $C_{m}=$ completeness, $C_{r}=$ correctness and $Q_{l}=$ quality, are for buildings of $50 \mathrm{~m}^{2}$ and $10 \mathrm{~m}^{2}$

\begin{tabular}{lcccccccccc}
\hline Areas & $C_{m p}$ & $C_{r p}$ & $Q_{l p}$ & $A_{o e}$ & $A_{c e}$ & $R M S E$ & $C_{m, 50}$ & $C_{r, 50}$ & $C_{m, 10}$ & $C_{r, 10}$ \\
\hline \hline AV & 88.85 & 96.71 & 86.25 & 11.14 & 3.28 & 0.58 & 91.0 & 96.71 & 89.32 & 96.71 \\
HB & 86.95 & 96.72 & 84.45 & 13.0 & 3.28 & 1.18 & 86.95 & 96.71 & 86.95 & 96.71 \\
\hline Avg & 87.90 & 96.72 & 85.35 & 12.07 & 3.28 & 0.8 & 88.97 & 96.71 & 88.14 & 96.71 \\
\hline \hline
\end{tabular}

Table 2. Building detection results: pixel-based evaluation for the Aitkenvale $(A V)$ and Hervey Bay $(H B)$ data sets in percentage. $C_{m p}=$ completeness, $C_{r p}=$ correctness and $Q_{l p}=$ quality, $A_{o e}=$ area omission error and $A_{c e}=$ area commission error in percentage, $R M S E=$ planimetric accuracy in metres

segmentation (last step), the NDVI value and average height of LiDAR points in that particular region, were laying well within the rule window that caused boundary to extend on tree bush and ground for buildings (h) and (i) respectively. The similar phenomenon can be observed from Figure (d) in 9(b), where part of bush was also included into the boundary due to similar height of building.

The buildings encircled with red ovals in Figure 8 (j)-(m) were not detected due to transparent roof material. These buildings have also been marked to their corresponding positions in Figure 8(a). These buildings were eliminated while generating primary building mask in first step because there were not LiDAR returns recorded for these buildings. But small sized umbrella $(\mathrm{k})$ was missed due to extremely small sized and misalignment between both input data sources. The buildings (n) and (o), marked with 'yellow' coloured oval in Figure 8(a), are the cases where building boundaries were not expanded towards building edges. The reason that actually stopped the boundary extension procedure in case of building (n) was a variation in LiDAR data height in the neighbourhood due to nearby vegetation. But the building (o) suffered merely because of misalignment between two data sources.

It is quite evident from Table 1 that the proposed technique has not only detected buildings larger than $50 \mathrm{~m}^{2}$ with $100 \%$ accuracy but has also extracted low height buildings with equal accuracy. As further can be noticed from Table 2, which tabulates pixel-based evaluation results, the average completeness, and quality values are even 2 to $4 \%$ higher than those in the object-based evaluation for the AV site. It can further be seen that our proposed algorithm offers an average accuracy of about $89 \%$ and $96 \%$ in pixel-based completeness and correctness for buildings larger than $50 \mathrm{~m}^{2}$, while at the same it gives an average accuracy of around $88 \%$ and $96 \%$ in pixel-based completeness and correctness for buildings smaller than $10 \mathrm{~m}^{2}$ respectively.

\subsection{COMPARATIVE PERFORMANCE}

The method proposed in this research outperforms the algorithm reported in (Awrangjeb and Fraser, 2014b) in completeness and correctness for both object-based and pixel-based evaluation. For the AV site, our proposed algorithm showed significantly better object-based accuracy than (Awrangjeb and Fraser, 2014b)-91\% vs $67 \%$, for the buildings smaller than $10 \mathrm{~m}^{2}$. Similarly, in terms of pixel-based accuracy, our proposed method offers $88 \%$ as compared to $86 \%$ of Awrangjeb's technique when all building objects are considered.

The visual comparative results of both detection techniques reported in (Awrangjeb and Fraser, 2014b) and proposed algorithm are shown in Figure 10 (a) and (b) respectively. We can clearly observe that Awrangjeb's algorithm fails to detect 11 buildings highlighted in red colour whereas the corresponding buildings detected by our proposed algorithm are shown in 10(b). Additionally, it can be noticed from building marked by yellow coloured oval in 10(a) that Awrangjeb's method fails to completely delineate the building, thus, indicate under-segmentation issue. But the corresponding yellow coloured oval in 10(b) shows that the proposed algorithm can successfully delineate the extended building parts efficiently.

But in case of the HB site, both the algorithms offer the same object-based accuracy for larger buildings. However, the pixelbased completeness of the proposed technique is $96 \%$ as of $93 \%$ by (Awrangjeb and Fraser, 2014b) for buildings larger than $50 \mathrm{~m}^{2}$.

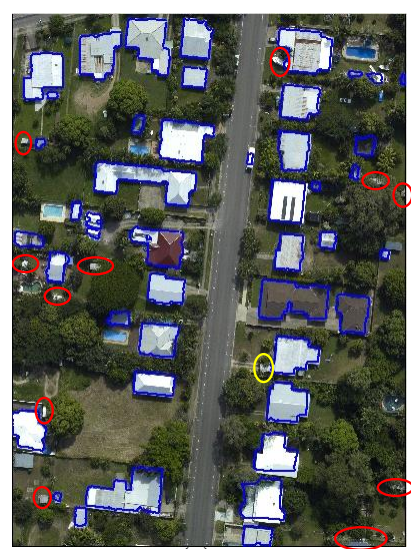

(a)

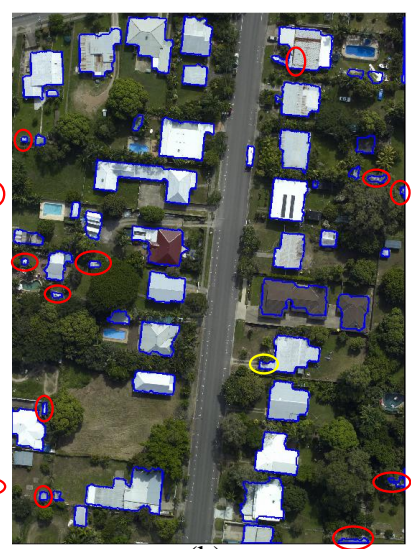

(b)
Figure 10. (a) Buildings missed by Awrangjeb(2014) detection technique, (b) Building detection result of proposed method

\section{CONCLUSION}

The automatic extraction of accurate building boundaries is an important geo-spatial information that is indispensable for several applications. The most challenging factor confronted in boundary delineation is building shape variability and surrounding environment complexity. In order to deal with various building types, this research presents a new method for automatic building detection through an effective integration of LiDAR data and multispectral imagery.

The proposed approach yields the complementary advantages from both the LiDAR data and multispectral image. The initial building positions are obtained after connected component analysis 
carried on primary building mask, derived from LiDAR data. Later on, several building features are extracted from multispectral image that are progressively used in different stages to eliminate false objects and vegetation, detect buildings, and delineate the corresponding building boundaries.

The final building boundary is obtained by extending the initial position using both the data sources: features extracted from image and LiDAR data. The whole procedure imposes no constraint on building shape variability and surrounding environment. This method is fully data-driven, self-adaptive and avoids the undersegmentation and over-segmentation issues. The proposed method is not only capable of detecting small buildings, but can also separate the buildings from surrounding dense vegetation and close buildings.

In future, the segmented lines extracted from multispectral imagery will be incorporated to obtain better planimetric accuracy and to generate the building footprints. This would help to develop 2D representation and reconstruction of roof features (e.g. roof planes, chimneys, and dorms) with the integration of LiDAR data. The accuracy and performance of the proposed approach will further be analysed on ISPRS benchmark data sets with different LiDAR resolutions.

\section{ACKNOWLEDGEMENTS}

The research is supported under the Discovery Early Career Researcher Award by the Australian Research Council (project number DE120101778). The Aitkenvale and Hervey Bay data sets were provided by Ergon Energy (http://www.ergon.com.au) in Queensland, Australia.

\section{REFERENCES}

Awrangjeb, M. and Fraser, C., 2014a. An automatic and threshold-free performance evaluation system for building extraction techniques from airborne lidar data. Selected Topics in Applied Earth Observations and Remote Sensing, IEEE Journal of 7(10), pp. 4184-4198.

Awrangjeb, M. and Fraser, C. S., 2014b. Automatic segmentation of raw lidar data for extraction of building roofs. Remote Sensing 6(5), pp. 3716-3751.

Awrangjeb, M., Ravanbakhsh, M. and Fraser, C. S., 2010. Automatic detection of residential buildings using lidar data and multispectral imagery. ISPRS Journal of Photogrammetry and Remote Sensing 65(5), pp. 457-467.

Awrangjeb, M., Zhang, C. and Fraser, C. S., 2012. Building detection in complex scenes thorough effective separation of buildings from trees. Photogrammetric Engineering \& Remote Sensing 78(7), pp. 729-745.

Awrangjeb, M., Zhang, C. and Fraser, C. S., 2013. Automatic extraction of building roofs using lidar data and multispectral imagery. ISPRS Journal of Photogrammetry and Remote Sensing 83(9), pp. 1-18.

Chen, L. and Zhao, S., 2012. Building detection in an urban area using lidar data and quickbird imagery. International Journal of Remote Sensing 33(15), pp. 5135-5148.

Demir, N., Poli, D. and Baltsavias, E., 2009. Extraction of buildings using images \& lidar data and a combination of various methods. International Archives of the Photogrammetry, Remote Sensing and Spatial Information Sciences 38(part 3/W4), pp. 7176.
Elberink, S., 2008. Problems in automated building reconstruction based on dense airborne laser scanning data. International Archives of the Photogrammetry, Remote Sensing and Spatial Information Sciences 37(part B3), pp. 93-98.

Gonzalez, R. C., Woods, R. E. and Eddins, S. L., 2003. Digital image processing using matlab. Eddins, S.L.,Prentice Hall, New Jersey.

Khoshelham, K., Nedkov, S. and Nardinocchi, C., 2008. A comparison of bayesian and evidence-based fusion methods for automated building detection in aerial data. International Archives of the Photogrammetry, Remote Sensing and Spatial Information Sciences 37(part B7), pp. 1183-1188.

Lee, D., Lee, K. and Lee, S., 2008. Fusion of lidar and imagery for reliable building extraction. Photogrammetric Engineering \& Remote Sensing 74(2), pp. 215-226.

Li, Y. and Wu, H., 2013. An improved building boundary extraction algorithm based on fusion of optical imagery and lidar data. Optik 124(22), pp. $5357-5362$.

Mayer, H., 1999. Automatic object extraction from aerial imagery - a survey focusing on buildings. Computer Vision and Image Understanding 74(2), pp. 138-149.

Rottensteiner, F., Trinder, J., Clode, S. and Kubik, K., 2003. Building detection using lidar data and multispectral images. 2, pp. 673-682.

Rottensteiner, F., Trinder, J., Clode, S. and Kubik, K., 2005. Using the dempstershafer method for the fusion of lidar data and multi-spectral images for building detection. Information Fusion 6(4), pp. 283-300.

Rottensteiner, F., Trinder, J., Clode, S. and Kubik, K., 2007. Building detection by fusion of airborne laser scanner data and multi-spectral images : Performance evaluation and sensitivity analysis. ISPRS Journal of Photogrammetry and Remote Sensing 62(2), pp. 135-149.

Rutzinger, M., Rottensteiner, F. and Pfeifer, N., 2009. A comparison of evaluation techniques for building extraction from airborne laser scanning. IEEE Journal of Selected Topics in Applied Earth Observations and Remote Sensing 2(1), pp. 11-20.

Sampath, A. and Shan, J., 2007. Building boundary tracing and regularization from airborne lidar point clouds. Photogrammetric Engineering \& Remote Sensing 73(7), pp. 805-812.

Sohn, G. and Dowman, I., 2007. Data fusion of high-resolution satellite imagery and lidar data for automatic building extration. ISPRS Journal of Photogrammetry and Remote Sensing 62(1), pp. 43-63.

Vu, T., Yamazaki, F. and Matsuoka., M., 2009. Multi-scale solution for building extraction from lidar and image data. International Journal of Applied Earth Observation and Geoinformation 11(4), pp. 281-289.

Yong, L. and Huayi, W., 2008. Adaptive building edge detection by combining lidar data and aerial images. International Archives of the Photogrammetry, Remote Sensing and Spatial Information Sciences 37(Part B1), pp. 197-202. 